\title{
DeepHateExplainer: Explainable Hate Speech Detection in Under-resourced Bengali Language
}

\author{
Md. Rezaul Karim \\ Fraunhofer FIT \& RWTH Aachen \\ University, Aachen, Germany \\ Sagor Sarker \\ Begum Rokeya University \\ Rangpur, Bangladesh
Bharathi Raja Chakravarthi
National University of Ireland, \\ Galway, Ireland
}

\author{
Sumon Kanti Dey \\ Noakhali Science and Technology \\ University, Bangladesh \\ Mehadi Hasan Menon \\ Begum Rokeya University \\ Rangpur, Bangladesh \\ Md. Azam Hossain \\ Islamic University of Technology \\ Gazipur, Bangladesh
}

\author{
Tanhim Islam \\ RWTH Aachen University \\ Aachen, Germany \\ Kabir Hossain \\ The University of Alabama \\ Tuscaloosa, USA \\ Stefan Decker \\ Fraunhofer FIT \& RWTH Aachen \\ University, Aachen, Germany
}

\begin{abstract}
The exponential growths of social media and micro-blogging sites not only provide platforms for empowering freedom of expressions and individual voices, but also enables people to express anti-social behavior like online harassment, cyberbullying, and hate speech. Numerous works have been proposed to utilize textual data for social and anti-social behavior analysis, by predicting the contexts mostly for highly-resourced languages like English. However, some languages are under-resourced, e.g., South Asian languages like Bengali, that lack computational resources for accurate natural language processing (NLP). In this paper ${ }^{1}$, we propose an explainable approach for hate speech detection from the under-resourced Bengali language, which we called DeepHateExplainer. Bengali texts are first comprehensively preprocessed, before classifying them into political, personal, geopolitical, and religious hates using a neural ensemble method of transformer-based neural architectures (i.e., monolingual Bangla BERT-base, multilingual BERT-cased/uncased, and XLM-RoBERTa). Important (most and least) terms are then identified using sensitivity analysis and layer-wise relevance propagation (LRP), before providing human-interpretable explanations ${ }^{2}$ for the hate speech detection. Finally, we compute comprehensiveness and sufficiency scores to measure the quality of explanations w.r.t faithfulness. Evaluations against machine learning (linear and tree-based models) and neural networks (i.e., CNN, Bi-LSTM, and Conv-LSTM with word embeddings) baselines yield F1-scores of $78 \%, 91 \%, 89 \%$, and $84 \%$, for political, personal, geopolitical, and religious hates, respectively, outperforming both ML and DNN baselines.
\end{abstract}

\section{KEYWORDS}

Hate speech detection, Under-resourced language, Bengali, Multimodal memes, Embeddings, Transformers, Interpretability.

\section{INTRODUCTION}

Exponential growths of micro-blogging sites and social media not only empower freedom of expressions and individual voices, but

\footnotetext{
1 Proceeding of IEEE International Conference on Data Science and Advanced Analytics (DSAA'2021), October 6-9, 2021, Porto, Portugal. ${ }^{2}$ To foster reproducible research, we make available the data, source codes, models, and notebooks: https://github.com/rezacsedu/DeepHateExplainer
}

also enables people to express anti-social behavior [1,2], such as cyberbullying, online rumours, and spreading hatred statements [3, 2]. Abusive speech expressing prejudice towards a certain group is also very common [2], and based on race, religion, and sexual orientation is getting pervasive. United Nations Strategy and Plan of Action on Hate Speech [4] defines hate speech as any kind of communication in speech, writing or behaviour, that attacks or uses pejorative or discriminatory language regarding a person or a group based on their religion, ethnicity, colour, gender or other identity factors. Bengali is spoken by 230 million people in Bangladesh and India [5], making it one of the major languages in the world. Although Bengali is a rich language with a lot of diversity, it is severely low-resourced for natural language processing (NLP). This is mainly due to the lack of necessary computational resources such as language models, labelled datasets, and efficient machine learning (ML) methods for various NLP tasks. Similar to other major languages like English, the use of hate speech in Bengali is also getting rampant, which is due to unrestricted access and use of social media and digitalization [6].

Some examples of Bengali hate speech and their respective English translations are shown in Fig. 1 that are either directed towards a specific person or entity or generalized towards a group. These examples signify how severe Bengali hateful statements could be. Nevertheless, there is a potential chance that these could lead to serious consequences such as hate crimes [2], regardless of languages, geographic locations, or ethnicity. Automatic identification of hate speech and raising public awareness is a non-trivial task [2]. However, manually reviewing and verifying a large volume of online content is not only time-consuming but also labor-intensive [7]. Further, accurate identification requires automated and robust ML methods. Compared to traditional ML and neural networks (DNNs)based approaches, state-of-the-art (SotA) language models are becoming increasingly effective. Nevertheless, a serious drawback of many existing approaches is that the outputs can neither be traced back to the inputs, nor it is clear why outputs are transformed in a certain way. This makes even the most efficient language models black-box methods. Therefore, how a prediction is made by an algorithm should be as transparent as possible to users to gain human trust in AI systems. 


\begin{tabular}{|c|c|c|}
\hline Statement & English translation & Context \\
\hline $\begin{array}{l}\text { • মালাউনের বাচ্চাদের বাংলাদেশে কোন স্থান নেই। } \\
\text { • মুসলমানরা আল কায়েদা, তালেবান এবং জঙ্গি। }\end{array}$ & $\begin{array}{l}\text { - Hindus have no place in Bangladesh. } \\
\text { - Muslims are Al-Qaeda, Taliban, and terrorists. }\end{array}$ & Religious \\
\hline $\begin{array}{l}\text { • কুন্তালীগের বাচ্চারা সন্ত্রাসী আর চেতনা ব্যবসায়ী। } \\
\text { • জামাত, শিবির, রাজাকার এই মুহৃর্তে বাংলা ছাড়। }\end{array}$ & $\begin{array}{l}\text { - Awami League is a terrorist organization and they are conscious businessmen. } \\
\text { - Anti-liberation war criminals should be deported from Bangladesh. }\end{array}$ & Political \\
\hline $\begin{array}{l}\text { • ঘরে ঢুকে মহিলাদের ধর্ষণ করে খুন করে দে। } \\
\text { • কুত্তার বাচ্চা হুই ড্রাগ আর মাগি নিয়েই পড়ে থাক। }\end{array}$ & $\begin{array}{l}\text { - Trespass each house and kill women after raping. } \\
\text { - You mother fucker live with drugs and whore. }\end{array}$ & Personal \\
\hline $\begin{array}{l}\text { • অবৈধ বাংলাদেশিদের অবিলস্বে ঘাড়ে ধাক্কা দিয়ে বিতাড়িত করা হবে। } \\
\text { • রেন্ডিয়ানরা বাংলাদেশিদের প্রধান শত্র৷। }\end{array}$ & $\begin{array}{l}\text { - Illegal Bangladeshis will be immediately pushed to the neck and be deported. } \\
\text { - Indians are the main enemies of Bangladesh. }\end{array}$ & Geopolitical \\
\hline
\end{tabular}

Figure 1: Example hate speech in Bengali, either directed towards a specific person or entity, or generalized towards a group

To mitigate the opaqueness of black-box models and inspired by recent successes of transformer language models (e.g., BERT [8], RoBERTa [9], XLNet [10], and ELECTRA [11]), we propose DeepHateExplainer - an explainable approach for hate speech detection from under-resourced Bengali language. Our approach is based on ensemble of BERT variants, including monolingual Bangla BERTbase [12], m-BERT (cased/uncased), and XLM-RoBERTa. Further, we provide global and local explanations of the predictions in a post-hoc fashion and measures of explanations w.r.t faithfulness.

\section{RELATED WORK}

Numerous works have been proposed to accurately and reliably identification of hate speech from major languages like English [1, 7]. Classic methods traditionally rely on manual feature engineering, e.g., support vector machines (SVM), Naïve Bayes (NB), logistic regression (LR), decision trees (DT), random forest (RF), and gradient boosted trees (GBT). On the other hand, DNN-based approaches that learn multilayers of abstract features from raw texts, are primarily based on convolutional (CNN) or long short-term memory (LSTM) networks. In comparison with DNNs, these approaches are rather incomparable as the efficiency of linear models at dealing with billions of such texts proven less accurate and unscalable. CNN and LSTM are two popular DNN architectures: CNN is an effective feature extractor, whereas LSTM is suitable for modelling orderly sequence learning problems. CNN extracts word or character combinations, e.g., n-grams, and LSTM learns long-range word or character dependencies in texts. While each type of network has relative advantages, several works have explored combining both architectures into a single network [13]. Conv-LSTM is a robust architecture to capture long-term dependencies between features extracted by CNN and found more effective than structures solely based on CNN or LSTM, where the class of a word sequence depends on preceding word sequences.

However, accurate identification of hate speech in Bengali is still a challenging task. Only a few restrictive approaches [14, 15, 2] have been proposed so far. Romim et al. [14] prepared a dataset of $30 \mathrm{~K}$ comments, making it one of the largest datasets for identifying offensive and hateful statements. However, this dataset has several issues. First, it is very imbalanced as the ratio of hate speech to nonhate speech is $10 \mathrm{~K}: 20 \mathrm{~K}$. Second, the majority of hate statements are very short in terms of length and word count compared to nonhate statements. Third, their approach exhibits a moderate level of effectiveness at identifying offensive or hateful statements, giving an accuracy of $82 \%$. Fourth, their approach is a black-box method. Ismam et al. [15] collected hateful comments from Facebook and annotated 5,126 hateful statements. They classified them into six classes- hate speech, communal attack, inciteful, religious hatred, political comments, and religious comments. Their approach, based on GRU-based DNN, achieved an accuracy of $70.10 \%$.

In a recent approach, Karim et al. [2], provided classification benchmarks for document classification, sentiment analysis, and hate speech detection for the Bengali language. Their approach, by combining fastText embeddings with multichannel Conv-LSTM network architecture, is probably the first work among a few other studies on hate speech detection. Their Conv-LSTM architecture, by combining fastText embeddings, outperformed Word2Vec and GloVe models, since fastText works well with rare words such that even if a word was not seen during the training, it can be broken down into n-grams to get its corresponding embeddings. All these restrictive approaches are black-box methods. On the contrary, interpretable methods put more emphasis on the transparency and traceability of opaque DNN models. With layer-wise relevance propagation (LRP) [16], relevant parts of inputs that caused a result can be highlighted [17]. To mitigate opaqueness and to improve explainability in hate speech identification, Binny et al. [18] proposed 'HateXplain' - a benchmark dataset for explainable hate speech detection. They observe that high classification accuracy is not everything, but high explainability is also desired. They measure the explainability of an NLP model w.r.t plausibility and faithfulness that are based on human rationales for training [19]. 


\section{PROPOSED APPROACH}

Inspired by SotA approaches and interpretability methods such as sensitivity analysis (SA) [20] and LRP [16], we propose DeepHateExplainer - a novel approach to accurate identification of hate speech in the Bengali. Bengali texts are first comprehensively preprocessed, before classifying them into political, personal, geopolitical, and religious hates, by employing an ensemble of different transformer-based neural architectures: monolingual Bangla BERT-base, multilingual BERT (mBERT)-cased/uncased, and XLMRoBERTa. Then, we identify important terms with SA and LRP to provide human-interpretable explanations, covering both global and local explainability. To evaluate the quality of explanations, we measure comprehensiveness and sufficiency. Further, we train several ML (i.e., LR, NB, KNN, SVM, RF, GBT) and DNN (i.e., CNN, Bi-LSTM, and Conv-LSTM with word embeddings) baseline models. To the end, DeepHateExplainer focuses on algorithmic transparency and explainability, with the following assumptions:

- A majority voting-based ensemble from a panel of independent NLP expert or linguists provides fairer and trustworthy prediction than a single expert.

- By decomposing the inner logic (e.g., what terms the model put more attention to) of a black-box model with probing and SA, the opaqueness can be reduced.

- By highlighting the most and least important terms, we can generate human-interpretable explanations.

Overall contributions of our approach are 4-folds:

(1) We prepared the largest hate speech detection dataset to date for the Bengali language.

(2) To the best of our knowledge, we are the first batch of researchers to employ neural transformer-based language models for hate speech detection for Bengali.

(3) We prepared several computational resources, such as annotated dataset, language models, source codes, and interpretability techniques that will further advance the NLP research for under-resourced Bengali language.

(4) We improved both local and global explainability and algorithmic transparency of black-box models by mitigating their opaqueness.

\section{DATASETS}

We extend the Bengali Hate Speech Dataset [2] with additional 5,000 labelled examples. The Bengali Hate Speech Dataset categorized observations into political, personal, geopolitical, religious, and gender abusive hates. However, based on our empirical study and linguist analysis, we observe that distinguishing personal from gender abusive hate is often not straightforward, as they often semantically overlap. To justify this, let consider example hate statements in Fig. 3. These statements (non-Bengali speakers are requested to refer to English translations) express hatred statement towards a person, albeit commonly used words such as খানকির বাচ্চা, শালী, পতিতা, নষ্টা, বেশ্যা, মাগি, খানকি, কুত্তার বাচ্চা (corresponding English terms are the girl of slut, slut, prostitute, fucking bitch, whore, waste, bitch), are directed mostly towards women. We follow a bootstrap approach for data collection, where specific types of texts containing common slurs and terms, either directed towards a specific person or entity or generalized towards a group, are only considered. Texts were collected from Facebook, YouTube comments, and newspapers. We categorize the samples into political, personal, geopolitical, and religious hate. Sample distribution and definition of different types of hates are outlined in Table 1.

\subsection{Data annotation}

Three annotators (a linguist, a native Bengali speaker, and an NLP researcher) participated in the annotation process. To reduce possible bias, unbiased contents are supplied to the annotators and each label was assigned based on a majority voting on the annotator's independent opinions. To evaluate the quality of the annotations and to ensure the decision based on the criteria of the objective, we measure inter-annotator agreement w.r.t Cohen's Kappa statistic [21]. Let consider $n$ target objects are annotated by $m(\geq 2)$ annotators into one of $k(\geq 2)$ mutually exclusive categories, the proportion of score $\bar{p}_{j}$ and the kappa $\hat{k}_{j}$ for category $j$ are computed as follows [21]:

$$
\begin{array}{r}
\bar{p}_{j}=\frac{\sum_{i=1}^{n} x_{i j}}{n m} \\
\hat{k}_{j}=1-\frac{\sum_{i=1}^{n} x_{i j}\left(m-x_{i j}\right)}{n m(m-1) \bar{p}_{j}\left(1-\bar{p}_{j}\right)},
\end{array}
$$

where $x_{i j}$ is possible scores on subject $i$ into category $j$. The overall kappa $\hat{\bar{k}}$ is subsequently computed as [21]:

$$
\hat{\bar{k}}=\frac{\sum_{j=1}^{k} \bar{p}_{j}\left(1-\bar{p}_{j}\right) \hat{k}_{j}}{\sum_{j=1}^{k} \bar{p}_{j}\left(1-\bar{p}_{j}\right)} .
$$

Taking into account the personal vs. gender abusive hate consideration, we observed a $\hat{\bar{k}}$ score of 0.87 , which is $3 \%$ of improvement over the previous approach by Karim et al. [2].

\section{METHODS}

In this section, we discuss our proposed approach in detail, covering word embeddings, network (ML/DNN/transformers) training, explanation generation, and measuring explainability.

\subsection{Data preprocessing}

We remove HTML markups, links, image titles, special characters, and excessive use of spaces/tabs, before initiating the annotation process. Further, following preprocessing steps are followed before training ML and DNN baseline models:

- Hashtags normalization: inspired by positive effects in classification task [22], hashtags were normalized.

- Stemming: inflected words were reduced to their stem, base or root form.

- Emojis and duplicates: all emojis, emoticons, duplicate, and user mentions were removed.

- Infrequent words: tokens with a document frequency less than 5 were removed.

However, as research has shown that BERT-based models perform better classification accuracy on uncleaned texts, we did not perform major preprocessing tasks, except for the lightweight preprocessing discussed above. 


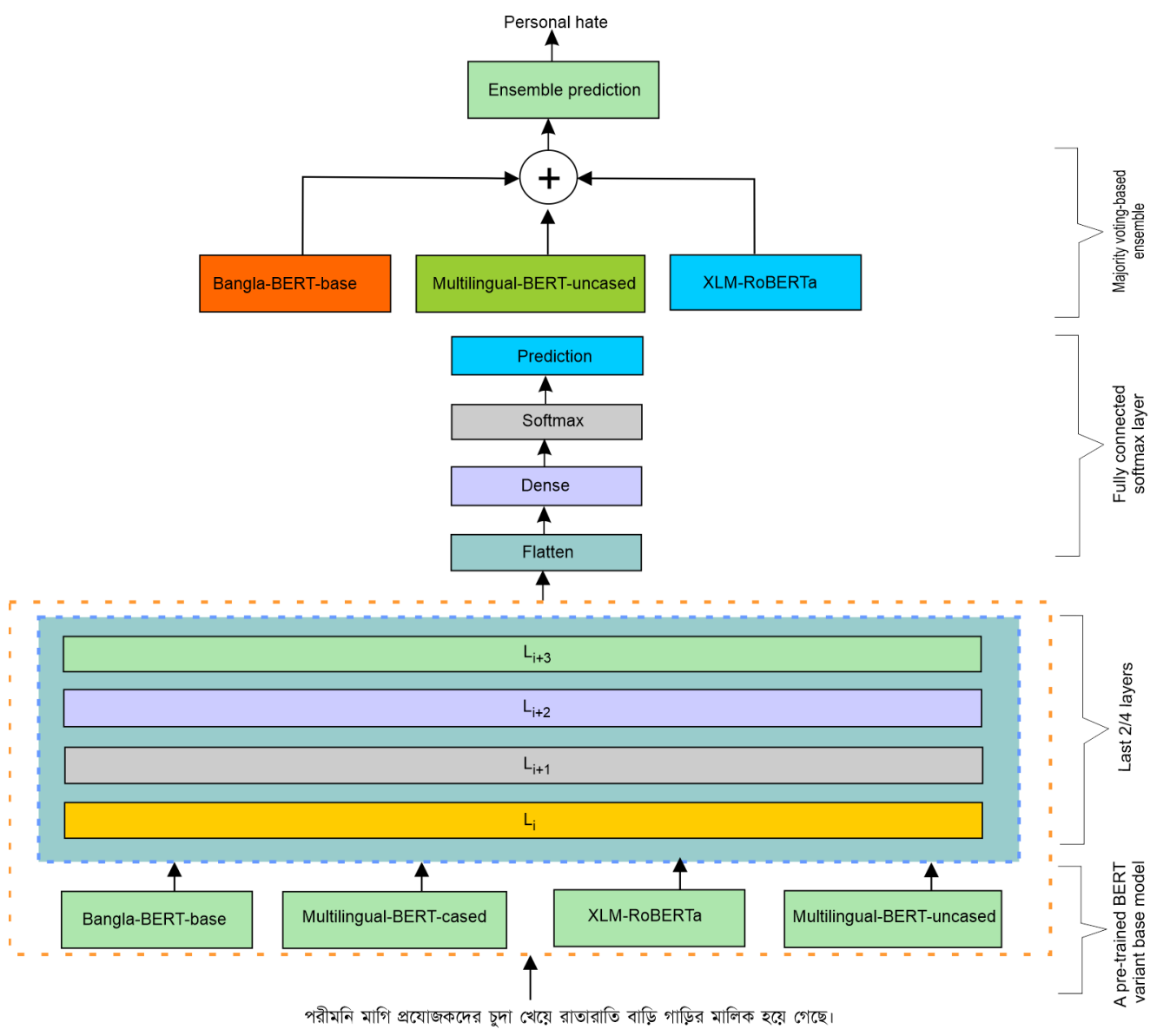

Figure 2: Schematic representation of proposed approach: each of 4 BERT variants is finetuned by adding a fully-connected softmax layer on top and cross-validation based on ensemble optimization, followed by majority voting ensemble

\begin{tabular}{|c|c|}
\hline Bengali hate statement & English translation \\
\hline খানকির বাচ্চাদের মৃত্যুদন্ড দেওয়া ভুল হয়েছে। ওদের সারা জীবন কষ্ট দিয়ে মারা উচিৎ ছিল। & It was wrong to execute them, the children of the prostitute should have died at the cost of their lives. \\
\hline প্রভা বাংলাদেশের সবচেয়ে বড় খানকি অভিনেন্রী। & Prova (Bangladeshi actress) is the worst slut in Bangladesh. \\
\hline তুই একটটা নষ্টা খানকির বাচ্চা, বেশ্যা খানা তোর জন্য উপযুক্ত জায়গা। & You're a fucking whore, brothel is the right place for you. \\
\hline পরীমনি মাগি প্রযোজকদের চুদা খেয়ে রাতারাতি বাড়ি গাড়ির মালিক বনে গেছে। & Porimoni (Bangladeshi actress) becomes owner of houses and cars overnight by giving fuck to producers. \\
\hline ঋতুপর্ণা একটটা অস্থির মান। মাগীটার বয়স যতো গড়াচ্েেহ ওর অভিনয় ও তত নোংরা হচ্েে। & Rituparna (Indian actress) is a fucking slut. The older this bitch gets, the more dirty her acting becomes. \\
\hline
\end{tabular}

Figure 3: Example hate statements directed towards a person, but may contextually be directed towards a women

Table 1: Statistics of the hate speech detection dataset

\begin{tabular}{l|l|l}
\hline Hate type & Description & \#Examples \\
\hline Political & Directed towards a political group/party & 999 \\
\hline Religious & Directed towards a religion/religious group & 1,211 \\
\hline Geopolitical & Directed towards a country/region & 2,364 \\
\hline Personal & Directed towards a person & 3,513 \\
\hline Total & & 8,087 \\
\hline
\end{tabular}




\subsection{Training of ML baseline models}

We train LR, SVM, KNN, NB, RF, and GBT ML baselines models ${ }^{3}$, using character n-grams and word uni-grams with TF-IDF weighting. The best hyperparameters are produced through random and with 5 -fold cross-validation tests.

\subsection{Neural word embeddings}

We train the fastText [23] word embedding model on Bengali articles used for the classification benchmark study by Karim et al. [2]. The preprocess reduces vocabulary size due to the colloquial nature of the texts and some degree, addresses the sparsity in the wordbased feature representations. We have also tested, by keeping word inflexions, lemmatization, and lower document frequencies. We observe slightly better accuracy using the lemmatization, which is the reason we reported the result based on it. The fastText model represents each word as an n-gram of characters, which helps capture the meaning of shorter words and allows the embeddings to understand suffixes and prefixes. Each token is embedded into a 300-dimensional real-valued vector, where each element is the weight for the dimension for the token. Since the annotated hate statements are relatively short, we constrain each sequence to 100 words by truncating longer texts and pad shorter ones with zero values to avoid padding in convolutional layers with many blank vectors for the majority of articles.

\subsection{Training of DNN baseline models}

We train three DNN baselines: CNN, Bi-LSTM, and Conv-LSTM. Weights of embedding layer for each network is initialized with the embeddings based on the fastText embedding model. Embedding layer maps each hate statement into a sequence (for LSTM and $\mathrm{CNN}$ layers) and transforms into feature representation, which is then flattened and feed into a fully connected softmax layer. Further, we add Gaussian noise and dropout layers to improve model generalization. AdaGrad optimizer is used to learn the model parameters by reducing the categorical-cross-entropy loss. We train each DNN architecture 5 separate times in a 5 -fold CV setting, followed by measuring the average macro $\mathrm{F} 1$-score on the validation set to choose the best hyperparameters ${ }^{4}$ using random search.

\subsection{Training of transformer-based models}

As shown in Fig. $2^{5}$, we train monolingual Bangla BERT-base, mBERT (cased and uncased), and XLM-RoBERTa large models. Bangla-BERT-base ${ }^{6}$ is a pretrained Bengali language model built with BERT-based mask language modelling. RoBERTa [9] is an improved variant of BERT, which is optimized by setting larger batch sizes, introducing dynamic masking, and training on larger datasets. XLM-RoBERTa [24] is a multilingual model trained on web crawled data. XLM-RoBERTa not only outperformed other transformer models on cross-lingual benchmarks but also performed better on various NLP tasks in a low-resourced language setting.

We shuffle training data for each epoch and apply gradient clipping. We set the initial learning rate to $2 e^{-5}$ and employ Adam

\footnotetext{
${ }^{3}$ Supplementary materials in arXiv version: https://arxiv.org/abs/2012.14353 ${ }^{4}$ Supplementary materials in arXiv version: https://arxiv.org/abs/2012.14353 ${ }^{5}$ English translation: Porimoni becomes the owner of houses and cars overnight after giving fuck to film producers. $\quad{ }^{6} \mathrm{https} / /$ huggingface.co/sagorsarker/bangla-bert-base
}

optimizer with the scheduled learning rate. Pre-trained BERT variants are fine-tuned by setting the maximum input length to 256 . We experimented with 2, 3, and 4 layers of multi-head attention, followed by a fully connected softmax layer. As we perform the ensemble of best models to report final predictions (as Fig. 4), several experiments with different hyperparameters combinations are carried out (Table 2), before saving the best epochs, for each model.

\subsection{Generating explanations}

We provide global and local explanations in a post-hoc fashion. For the former, a list of most and least relevant words for each class is identified based on linguist analysis. To provide overall global interpretability, feature importance (FI) is computed for model $f$. For feature $x_{i}$ in observation $x \in X$ and for each repetition $r$ in $1,2, \ldots, R$, column $x_{i}$ is randomly shuffled to generate a corrupted version $\tilde{X}_{r, x_{i}}$ for $X$. A reference balanced score $s_{r, x_{i}}$ is then computed for $f$. The mean importance $\sigma_{x_{i}}$ for feature $x_{i}$ is then computed as follows [25]:

$$
\sigma_{x_{i}}=s-\frac{1}{R} \sum_{r=1}^{R} s_{r, x_{i}} .
$$

For the latter, we identify which features in a sample are important for individual prediction. Relevance score (RS) as a measure of importance is computed with SA and relevance conservation LRP [25]. For input vector $x$, RS $R_{d}$ is computed for each input dimension $d$. This is analogous to quantify the relevance of $x_{d}$ w.r.t to target class $c$. Then the RS $R_{d}$ is generated by computing squared partial derivatives as [25]:

$$
R_{d}=\left(\frac{\partial f_{c}}{\partial x_{d}}(x)\right)^{2}
$$

where $f_{c}$ is a prediction score function for class $c$. Total relevances is then computed by summing relevances of all input space dimensions $d$ [25]:

$$
\left\|\nabla_{\boldsymbol{x}} f_{c}(x)\right\|_{2}^{2} \text {. }
$$

In contrast to SA, LRP is based on the layer-wise relevance conservation principle. LRP redistributes the quantity $f_{c}(x)$ from output layer to the input layer. The relevance for the output layer neuron is set to $f_{c}(x)$ w.r.t to the target class $c$, by ignoring irrelevant output layer neurons. The layer-wise relevance score for each intermediate lower-layer neuron is computed based on weighted connections. Assuming $z_{j}$ and $z_{i}$ are an upper-layer and a lower-layer neuron, respectively, and the value of $z_{j}$ is already computed in the forward pass as $\sum_{i} z_{i} \cdot w_{i j}+b_{j}$, where $w_{i j}$ and $b_{j}$ are the weight and bias, the relevance score $R_{i}$ for the lower-layer neurons $z_{i}$ is then computed by distributing the relevences onto lower-layer. The relevance propagation $R_{i \leftarrow j}$ from upper-layer neurons $z_{j}$ to lower-layer neurons $z_{i}$ is computed as a fraction of the relevance $R_{j}$. Subsequently, all the incoming relevance for each lower-layer neuron is summed up as [25]:

$$
R_{i \leftarrow j}=\frac{z_{i} \cdot w_{i j}+\frac{\epsilon \cdot \operatorname{sign}\left(z_{j}\right)+\delta \cdot b_{j}}{N}}{z_{j}+\epsilon \cdot \operatorname{sign}\left(z_{j}\right)} \cdot R_{j}
$$


Table 2: Hyperparameter combinations for training BERT variants

\begin{tabular}{l|l|l|l|l}
\hline Hyperparameter & Bangla-BERT & mBERT cased & mBERT-uncased & XLM-RoBERTa \\
\hline Learning-rate & $3 \mathrm{e}-5$ & $2 \mathrm{e}-5$ & $5 \mathrm{e}-5$ & $2 \mathrm{e}-5$ \\
Epochs & 6 & 6 & 6 & 5 \\
Max seq length & 128 & 128 & 128 & 128 \\
Dropout & 0.3 & 0.3 & 0.3 & 0.3 \\
Batch size & 16 & 16 & 16 & 16 \\
\hline
\end{tabular}

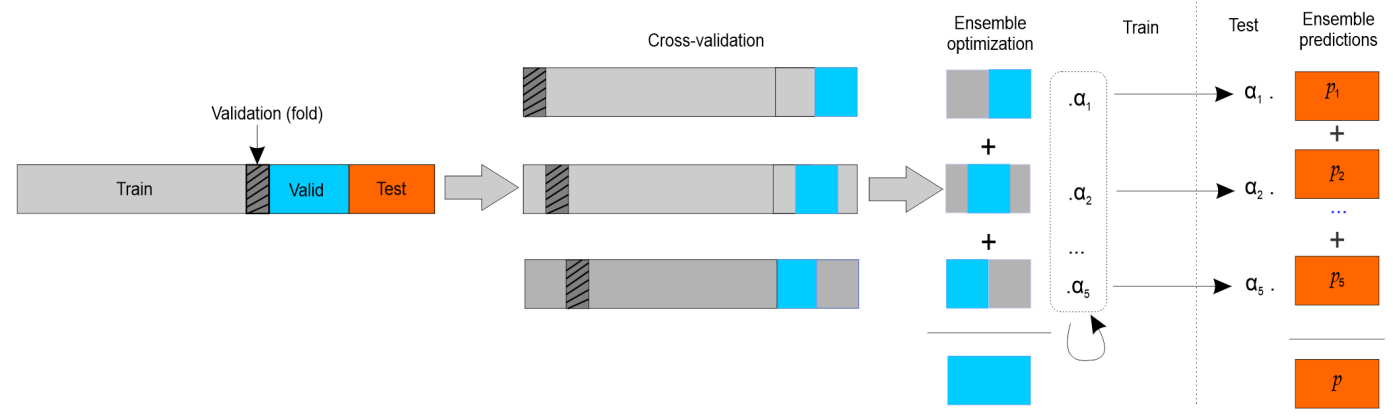

Figure 4: A representation of cross-validation (CV) ensemble optimization process. The final ensemble weights $\alpha_{1}, \alpha_{2}, \cdots, \alpha_{M}$ in which $\mathrm{M}$ is the number of $\mathrm{CV}$ folds used to combine model predictions and evaluate performance on test set

where $N$ is total number of lower-layer neurons connected to $z_{j}$, $\epsilon$ is a stabilizer, $\operatorname{sign}\left(z_{j}\right)=\left(1_{z_{j} \geq 0}-1_{z_{j}<0}\right)$ is the sign of $z_{j}$, and $\delta$ is a constant multiplicative factor set to 1 , to conserve the total relevance of all neurons in the same layer. Finally, $R_{i}$ is computed as $R_{i}=\sum_{j} R_{i \leftarrow j}$ [25].

\subsection{Measuring explainability}

System causability scale (SCS) [17] is proposed to measure the quality of explanations. SCS is based on the notion of causability and adapted from a usability scale and aims to determine whether and to what extent a user interface is explainable or which explanation process itself is suitable for the intended purpose [17]. Since SCS is based on usability feedback for an explainable interface, it is not suitable for our case. Therefore, we compute faithfulness w.r.t comprehensiveness and sufficiency to measure the quality of explanations based on ERASER [26]. To measure comprehensiveness, a contrast example $\tilde{x}_{i}$ is created, for each sample $x_{i}$, where $\tilde{x}_{i}$ is calculated by removing predicted rationales $r_{i}$ from $x_{i}$. Let $f\left(x_{i}\right)_{c}$ be the original prediction probability for model $f$ and for predicted class $c$. If model $f$ is defined as $f\left(x_{i} \backslash r_{i}\right)_{c}$ as the predicted probability of $\tilde{x}_{i}\left(=x_{i} \backslash r_{i}\right)$, it is expected that the prediction will be lower on removing the rationales [26]. The comprehensiveness metric $e$ is then calculated as follows [26]:

$$
e=f\left(x_{i}\right)_{c}-f\left(x_{i} \backslash r_{i}\right)_{c}
$$

The concept of rationales is proposed by Zaidan et al. [19] in NLP in which human annotators would highlight a span of text that could support their labelling decision, e.g., to justify why a review is positive, an annotator can highlight most important words and phrases that would tell someone to see the movie. To justify why a review is negative, highlight words and phrases that would tell someone not to see the movie. It is found to be useful in downstream NLP tasks like hate speech detection [18], text classification [27]. We conceptualize a similar idea w.r.t leave-one-feature-out analysis, where the rationale is computed based on the number of highlighted features divided by the number of features in a test sample. A prediction is considered a match if it overlaps with any of the ground truth rationales $r_{i} \geq 0.5$. A high value of comprehensiveness implies that the rationales were influential in the prediction. The sufficiency $s$, which measures the degree to which extracted rationales are adequate for the model $f$, which is measured as follows [26]:

$$
s=f\left(x_{i}\right)_{c} f\left(r_{i}\right)_{c}
$$

\section{RESULTS}

We discuss experimental results both qualitatively and qualitatively and explain the predictions globally and locally. Besides, we provide a comparative analysis with baselines.

\subsection{Experiment setup}

Programs were implemented using scikit-learn, Keras, and PyTorch and networks are trained on Nvidia GTX 1050 GPU. Open source implementation of fast Text ${ }^{7}$ is used to learn embeddings. $S H A P^{8}$ and $E L I 5^{9}$ are used to compute FI. Each model is trained on $80 \%$ of data, followed by evaluating the model on $20 \%$ held-out data. We report precision, recall, F1-score, and Matthias correlation coefficient (MCC). Finally, we perform the ensemble of top-3 models to report the final predictions. We select the best models with WeightWatcher ${ }^{10}$ [28]. Using WeightWatcher, the models giving the lowest log-norm and highest weighted-alpha are only considered. This is backed by the

\footnotetext{
7 https://radimrehurek.com/gensim/models/fasttext.html

${ }^{8}$ https://github.com/slundberg/shap $\quad 9$ https://github.com/eli5-org/eli5

${ }^{10}$ https://github.com/CalculatedContent/WeightWatcher
} 
fact that a lower log-norm signifies better generalization of network weights for unseen examples [28].

\subsection{Analysis of hate speech detection}

We evaluated 4 variants of BERT models on the held-out test set and report the results ${ }^{11}$ in Table 3. XML-RoBERTa model turns out to be both best performing and best-fitted model, giving the top F1-score of $87 \%$, which is about $2 \%$ to $5 \%$ better than other transformer models, while Bangla BERT-base and mBERT-uncased also performed moderately well. Based on metrics and the lowest log-norm, top-3 models were picked using WeightWatcher for the ensemble prediction, followed by discarding the mBERT-cased model from the voting ensemble. The highest MCC score of 0.82 is achieved with the ensemble prediction, which is slightly better than that of the XLM-RoBERTa, giving an MCC score of 0.808. Overall, MCC scores of $\geq 0.77$ were observed for each BERT-based model w.r.t Pearson correlation coefficient. This signifies that predictions are strongly correlated with ground truths and BERT variants are more effective compared to ML or DNN baseline models.

Confusion matrices in Fig. 5 show the breakdown of correct and incorrect classifications for each class, which correspond to ground truths vs. predicted labels. Ensemble prediction boosts the accuracy by at least $1.8 \%$ across the classes w.r.t F1-score, compared to top mBERT-cased and XML-RoBERTa models. Nevertheless, misclassification rates for all the classes have reduced significantly and overall 21 observations were correctly classified. This improvement signifies, to large extent, that ensemble prediction is effective at minimizing confusions. Further, as classes are imbalanced, accuracy alone gives a distorted estimation of the performance. Thus, we provide class-specific classification reports in Table 4 based on the ensemble prediction. Overall, our approach identifies personal hates more accurately compared to other types of hate w.r.t F1score. Identifying political hate was more challenging (giving an F1-score of 0.78 ) as political hates contain some terms that are often used to express personal hates.

\subsection{Comparison with baselines}

Since efficient feature selection can have significant impacts on model performance for ML methods [2], we observe the performance with manual feature selection. Forests of trees concept ${ }^{12}$ is employed to compute impurity-based FI. Each model is then trained by discarding irrelevant features. The feature selection helped SVM, $\mathrm{KNN}, \mathrm{RF}$, and GBT models improve their accuracy. GBT model performs the best among all ML baseline models, giving an MCC score of 0.571, albeit F1-scores for both RF and GBT are equal. RF model performs reasonably well, giving an F1-score of $68 \%$. Contrarily, performance of SVM, LR, and NB classifiers degraded significantly. LR model is not resilient to class discriminating features that could be lost during the feature selection, perhaps the conditional independence assumption (where features are assumed to be independent when conditioned upon class labels) of NB is not hold. Overall, the performance of each ML baseline model was severely poor, making them not suitable for reliable identification of hate statements.

\footnotetext{
11 Based on hyperparameter combinations in Table 2. $\quad{ }^{12}$ Forests of trees concept is a meta-transformer for selecting features w.r.t importance weights.
}

Each DNN baseline model is evaluated by initializing the embedding layer's weight with fastText embeddings. As observed, each model either outperforms or gives comparable performance to ML baseline models. In particular, Conv-LSTM performs the best among DNN baselines, giving F1 and MCC scores of 0.78 and 0.694 , respectively, which is about 4 to $5 \%$ better than Bi-LSTM (the second-best DNN baseline) and GBT (the best among ML baseline) models, respectively; while the F1-scores for CNN and Bi-LSTM reached to 0.73 and 0.75 , respectively, making them comparable to GBT and RF models. Overall, DNN baseline models also performed poorly compared to transformer-based models (ref. Table 3), albeit the fastText embedding model could have captured the word-level semantics sufficiently.

\subsection{Explaining hate speech detection}

We provide both local and global explanations for hate speech identification. For the former, we highlight globally important terms. Fig. 6 shows most frequently used terms expressing hatred statements (English terms: Rajakar, war criminals, Muslim, militant, Hindu, Jihadi ${ }^{13}$, Rohinga ${ }^{14}$, Pakistanis, Indians, Bangladesh Jamaate-Islami ${ }^{15}$, war criminals, whore, fuck, ass, rape, execution, Kutta League $^{16}$, consciousness $^{17}$, Hammer League ${ }^{18}$, son of a pig, slut, bastard, son of a bitch, broker ${ }^{19}$ ). These findings are further validated with the linguistic analysis, outlining the semantic meaning and relevance of these words. The most and least SA- and LRPrelevant word lists for each class are shown in Fig. 8 and Fig. 7, respectively that are used to express hatred statements.

Local explanations for individual samples are provided by highlighting the most important terms. We provide class-wise example heat maps based on SA and LRP-based relevances in Fig. 9 exposing different types of hates, where the colour intensity is normalized to the maximum relevance per hate statement. To quantitatively validate the word-level relevances for local explainability, we perform the leave-one-out experiment - we aim to improve the greedy backward elimination algorithm by preserving more interactions among terms. First, we randomly select a sample hate statement (e.g., same as Fig. 9b) in the test set. Then, we generate prediction probabilities for all the classes, followed by explaining word-level relevance for the two highest probable classes.

Let consider the example in Fig. 10: words on the right side are positive, while words on the left are negative. Words like জাতি, দখলে, and হিন্দ্ম (race Occupy, and Hindu in English, respectively) are positive for religious class, albeit the most significant word জাতি (race in English) is negative for personal hate category (where words বাচ্চারা and হারামজাদা (son of a bitch and bastard in English) are more important). Word জাতি has the highest positive score of 0.27 for class religious. Our model predicts this as a religious hate statement too, with the probability

\footnotetext{
13 Term to accuse Muslims to be terrorist in India, Pakistan, and Bangladesh. ${ }^{14}$ People who flew from genocide and ethnic cleansing by the Myanmar army and got asylum in Bangladesh. ${ }^{15}$ Islamist political party in Bangladesh. ${ }^{16}$ Hatred term for student organization of Bangladesh Awami League, where Kutta means dogs. ${ }^{17}$ The hatred form for Bangladesh Awami League, whose political agenda is backed by liberation war. 18 The hatred term of the student league - the official student organization of Bangladesh Awami League, who are suspects of killing many oppositions and innocent people with a hammer and hock-stick. ${ }^{19}$ Supporters of Bangladesh Awami League are called brokers of India, while supporters of Bangladesh Nationalist Party and Bangladesh Jamaat-e-Islami are called brokers of Pakistan.
} 
Table 3: Performance of hate speech detection

\begin{tabular}{c|l|l|l|l|l}
\hline Method & Classifier & Precision & Recall & F1 & MCC \\
\hline \multirow{5}{*}{ ML baselines } & LR & 0.68 & 0.68 & 0.67 & 0.542 \\
& NB & 0.65 & 0.65 & 0.64 & 0.511 \\
& SVM & 0.67 & 0.67 & 0.66 & 0.533 \\
& KNN & 0.67 & 0.67 & 0.66 & 0.533 \\
& RF & 0.69 & 0.69 & 0.68 & 0.561 \\
& GBT & $\mathbf{0 . 7 1}$ & $\mathbf{0 . 6 9}$ & $\mathbf{0 . 6 8}$ & $\mathbf{0 . 5 7 1}$ \\
\hline \multirow{5}{*}{ DNN baselines } & CNN & 0.74 & 0.73 & 0.73 & 0.651 \\
& Bi-LSTM & 0.75 & 0.75 & 0.75 & 0.672 \\
& Conv-LSTM & $\mathbf{0 . 7 9}$ & $\mathbf{0 . 7 8}$ & $\mathbf{0 . 7 8}$ & $\mathbf{0 . 6 9 4}$ \\
\hline \multirow{5}{*}{ BERT variants } & Bangla BERT & 0.86 & 0.86 & 0.86 & 0.799 \\
& mBERT-cased & 0.85 & 0.85 & 0.85 & 0.774 \\
& XML-RoBERTa & $\mathbf{0 . 8 7}$ & $\mathbf{0 . 8 7}$ & $\mathbf{0 . 8 7}$ & $\mathbf{0 . 8 0 8}$ \\
& mBERT-uncased & 0.86 & 0.86 & 0.86 & 0.795 \\
& Ensemble* & $\mathbf{0 . 8 8}$ & $\mathbf{0 . 8 8}$ & $\mathbf{0 . 8 8}$ & $\mathbf{0 . 8 2 0}$ \\
\hline
\end{tabular}

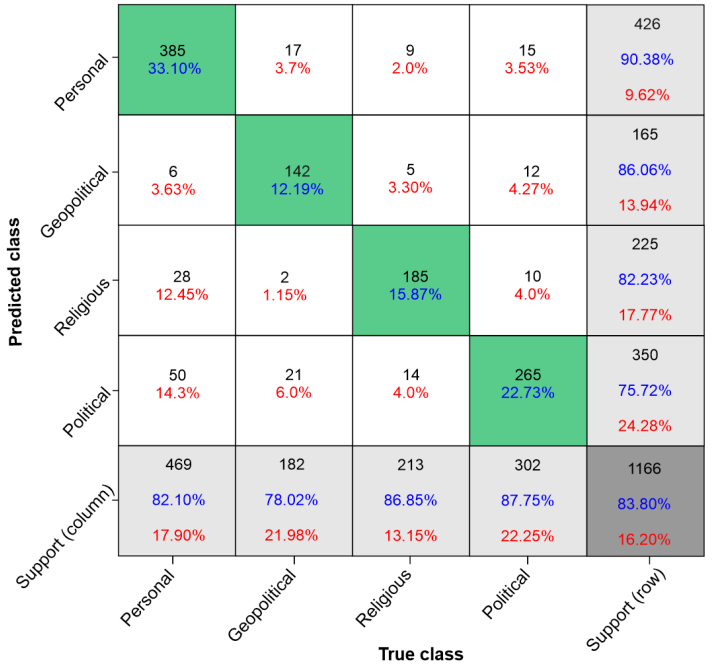

(a) For standalone XLM-RoBERTa

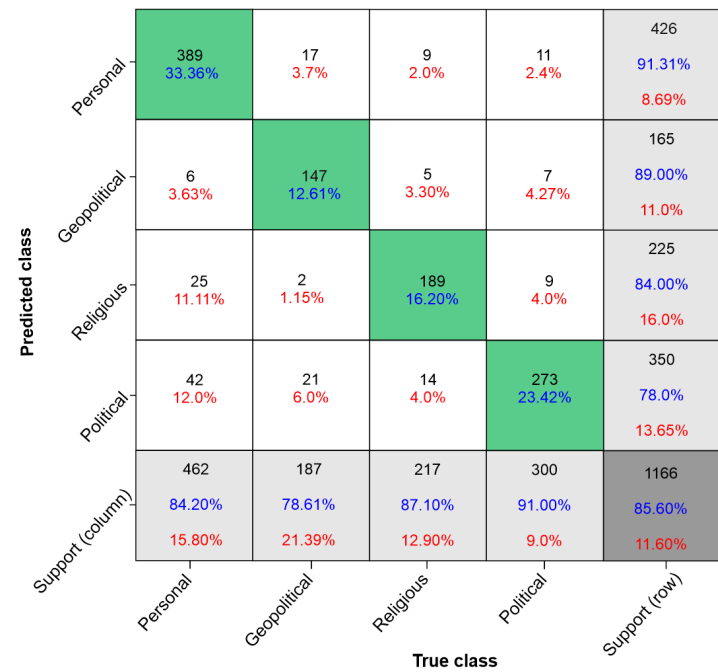

(b) For ensemble prediction

Figure 5: Confusion matrices: standalone XLM-RoBERTa vs. ensemble prediction (color code: red, blue, and black indicate misclassification rates, correct classification rates (in \%), and count, respectively)

রাজাকার, মুসলিম, জঙ্গি, মালাডন, জিহাদি, রোহিঙ্গা, পাকি, রেন্ডিয়া, জামাত, শিবির, যুদ্ধোপরাধী, বেশ্যা, চুদ, গুদ, ধর্ষণ, ফাঁসি, কুত্তালীগ, চেতনাবাজ, হাতুড়িলীগ, শুওেরের বাচ্চা, মাগি, হারামি, কুত্তারবাচ্চা, দালাল

Figure 6: Globally most important terms that are used to express hatred statements for all the hate classes

Table 4: Class-wise classification report based on majority voting ensemble of top-3 classifiers

\begin{tabular}{l|l|l|l}
\hline Hate type & Precision & Recall & F1 \\
\hline Personal & 0.91 & 0.90 & 0.91 \\
Political & 0.82 & 0.74 & 0.78 \\
Religious & 0.79 & 0.90 & 0.84 \\
Geopolitical & 0.89 & 0.89 & 0.89 \\
\hline
\end{tabular}

of 59\%. However, if we remove word জाতি from the text, we would expect the model to predict the label religious with a probability of $32 \%$ (i.e., $59 \%-27 \%$ ). Word জाTि is negative for personal hate category, albeit words বাচ্চারা and হারামজাদা have positive scores of 0.23 and 0.17 for the class personal. These identified words not only reveals the relevance of important terms for classifier's decision, but also signify that removing most relevant terms will impact the final decision, accordingly to their relevance value.

\subsection{Measure of explainability}

For measuring the explainability, only top models (ML, DNN, and BERT variants) are considered based on the results we analyzed 


\begin{tabular}{|c|c|c|c|}
\hline \multirow[b]{2}{*}{ Religious } & Most relevant & $\begin{array}{l}\text { ইসলাম, মালাডন, হিন্দ্র, মুসলিম, জঙ্গি, হুজুর, মসজিদ, } \\
\text { মন্দির, মাল, মূর্তিপুজা, মাদ্রাসা, আরব, তেতুল হুজুর, } \\
\text { জিহদ্দি, কোরআন, খলিফা, হুর, জাহন্নাম, নরক, বাইবেল, } \\
\text { শিবির, জামাত। }\end{array}$ & $\begin{array}{l}\text { Islam, Malaun, Hindu, militant, Lord, mosque, temple, Malu, } \\
\text { idolatry, madrasa, Arab, Tantul Huzur, Jihadi, Quran, Caliph, } \\
\text { Whore, Hell, Abyss,Bible, Shibir, Jamaat. }\end{array}$ \\
\hline & Least relevant & $\begin{array}{l}\text { বিবি, সহবস্থান, দৃর্গাপৃজা, কালীপৃজা, ঈদ, তারাবি, রোজা, } \\
\text { হজজ্, নামাজ। }\end{array}$ & $\begin{array}{l}\text { Wife, coexistence, Durga Puja (main religious festival of Hindus } \\
\text { in Bangladesh and West Bengal, India), Kali Puja (the second } \\
\text { main religious festival of Hindus in Bangladesh), Eid, Tarabi } \\
\text { (prayers performed by Muslim at night during Ramadan), } \\
\text { fasting, Hajj (pilgrim by Muslim), prayers. }\end{array}$ \\
\hline \multirow[t]{2}{*}{ Personal } & Most relevant & 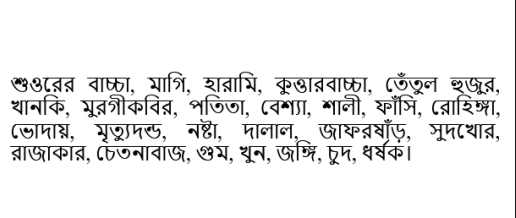 & $\begin{array}{l}\text { Swine, prostitute, bastard, young dog, Tamarind lord, whore, } \\
\text { Chicken Kabir (the hatred name of activist Shahriar Kabir who } \\
\text { is known as a chicken supplier to Pakistan army during the } \\
\text { liberation war in 1971), prostitute, whore, usurer, death by } \\
\text { execution, Rohingya, vagina, death penalty, slut, broker, Jafar } \\
\text { bull (the hatred name of famous writer Professor Jafar Iqbal in } \\
\text { Bangladesh), usurer, Razakar, conscious, kidnapping, murder, } \\
\text { militant, fuck, rapist. }\end{array}$ \\
\hline & Least relevant & $\begin{array}{l}\text { মানুষ, কলঙ্ক, তালেবান, মহিলা, চেয়েরা, পিতা, সন্তান, } \\
\text { আমানত, ব্যাঙ্ক, টাকা, ডাকাত, চোর, বাটপার, ইসলামী } \\
\text { ব্যাংক। }\end{array}$ & $\begin{array}{l}\text { Human, stigma, Taliban, female, girls, father, children, deposits, } \\
\text { banks, money, robbers, thieves, cheater, Islami bank. }\end{array}$ \\
\hline \multirow[t]{2}{*}{ Geopolitical } & Most relevant & $\begin{array}{l}\text { পাকিস্তান, ভারত, রেল্ডিয়া, বাংলাদেশ, মিয়ানমার, রোহিঙ্গা, } \\
\text { ব্রিটিশ, আমেরিকান, ফিলিস্তিন, কলকাতা, ট্রাম্প, ঢাকাইয়া, } \\
\text { ফাকিস্তান, ইসররাইন, টার্কি, আমিরাত, আরব, কাংলাদেশ, } \\
\text { পাকি, মোদী, বিজেপি, ইন্ডিয়া, ইরাক। }\end{array}$ & $\begin{array}{l}\text { Pakistan, India, Rendia (hatred form of India by Bangladeshis), } \\
\text { Bangladesh, Myanmar, Rohingya, British, American, Palestine, } \\
\text { Kolkata, Trump, Dhaka, Pakistan, Israel, Turkey, UAE, Arab, } \\
\text { Kangladesh (hatred form of Bangladesh by Indians), Paki (the } \\
\text { hatred form for Pakistanis), Modi, BJP, India, Iraq. }\end{array}$ \\
\hline & Least relevant & $\begin{array}{l}\text { ট্রানজিট, ফারাক্কা, চীন, হাসিনা, সীমান্ত, সুন্দরবন, কাশ্মীর, } \\
\text { বিএসএফ। }\end{array}$ & $\begin{array}{l}\text { Transit, Farakka, China, Hasina, border, Sundarbans (largest } \\
\text { mangrove forest in Bangladesh and India), Kashmir, BSF. }\end{array}$ \\
\hline
\end{tabular}

Figure 7: Globally most important terms used to express hatred statements for each hate class and their relevance interpretation

in Section 6.2 and Section 6.3. Results of the faithfulness in terms of comprehensiveness and sufficiency are shown in Table 5. As shown, XML-RoBERTa attained the highest comprehensiveness and sufficiency scores, outperforming other standalone models. Overall, BERT variants not only attained higher scores but also consistently outperforms other models such as GBT and Conv-LSTM baselines. Further, our study outlines two additional observations:

(1) GBT model shows both higher comprehensiveness and sufficiency compared to Conv-LSTM model, albeit the latter outperformed the former in classification task w.r.t classification metrics.

(2) As for BERT variants, Bangla BERT and mBERT-cased generate the least faithful explanations.

This signifies that a model that attains the best scores w.r.t metrics, may not perform well in terms of faithfulness explainability metrics. Based on this observation, it would not be unfair to say that a model's performance metric alone is not enough as models with slightly lower performance, but much higher scores for
Table 5: Measure of explainability

\begin{tabular}{l|c|c}
\hline Classifier & Comprehensiveness & Sufficiency \\
\hline GBT & 0.79 & 0.25 \\
\hline Conv-LSTM & 0.73 & 0.15 \\
\hline Bangla BERT & 0.78 & 0.25 \\
\hline XML-RoBERTa & 0.84 & 0.44 \\
\hline mBERT-uncased & 0.81 & 0.35 \\
mBERT-cased & 0.76 & 0.28 \\
\hline
\end{tabular}

faithfulness might be preferred for sensitive use cases such as hate speech detection at hand.

\section{CONCLUSION}

In this paper, we proposed DeepHateExplainer - an explainable approach for hate speech detection for under-resourced Bengali language. Based on ensemble prediction, DeepHateExplainer can detect different types of hates with an F1-score of $88 \%$, outperforming several ML and DNN baselines. Our study suggests that: i) feature 


\begin{tabular}{|c|c|c|c|c|c|c|c|}
\hline \multicolumn{2}{|c|}{ Religious: top features } & \multicolumn{2}{|c|}{ Geopolitical: top features } & \multicolumn{2}{|c|}{ Political: top features } & \multicolumn{2}{|c|}{ Personal: top features } \\
\hline Weight & Feature & \multirow{18}{*}{$\begin{array}{r}\text { Weight } \\
+1.702 \\
+0.825 \\
+0.798 \\
+0.786 \\
+0.779 \\
+0.773 \\
+0.729 \\
+0.724 \\
+0.610 \\
+0.550 \\
+0.430 \\
+0.420 \\
+0.420 \\
+0.410 \\
+0.420 \\
+0.420 \\
+0.410 \\
+0.320\end{array}$} & Feature & Weight & Feature & Weight & Feature \\
\hline+1.991 & शिन्दू & & পাকিস্তান & +2.016 & কুও্তালীগ & +1.193 & শওরেরবাচ্চা \\
\hline+1.925 & জজ্গি & & ভারত & +1.951 & রাজাকার & +1.030 & মাগি \\
\hline+1.834 & হুজুর & & রেল্ডিয়া & +1.758 & চে৩নাবাজ & +1.021 & হারামি \\
\hline $\begin{array}{l}+1.813 \\
+1697\end{array}$ & $\begin{array}{l}\text { মসাজদ } \\
\text { মन्দির }\end{array}$ & & বাংলাদেশ & $\begin{array}{l}+1.697 \\
+1655\end{array}$ & $\begin{array}{l}\text { श|ूুড়লাগ } \\
\text { পলिশলীগ }\end{array}$ & +0.946 & কুসারবাচ্চা \\
\hline $\begin{array}{l}+1.697 \\
+1.696\end{array}$ & মৃর্তিপূজা & & $\begin{array}{l}\text { |ময়ান মার } \\
\text { রোহিঙ্গা }\end{array}$ & $\begin{array}{l}+1.005 \\
+1.522\end{array}$ & তারেকজিয়া & $\begin{array}{l}+0.899 \\
+0.797\end{array}$ & $\begin{array}{l}\text { তেতুলছজজুর } \\
\text { খানকি }\end{array}$ \\
\hline+1.617 & মाদ্রাসা & & ব্রিটিশ & +1.518 & খালেদ & +0.700 & মুরগীকবির \\
\hline+1.594 & আরব & & আমেরিকান & +1.516 & জামাত & +0.640 & ফাঁসम \\
\hline+1.497 & তেঁতুলহুজুর & & ফিनिস্তিন & +1.420 & শিশবির & +0.550 & রোহিঙ্গা \\
\hline+1.488 & জিহাদি & & কলকাতা & +1.320 & $\begin{array}{l}\text { যুদ্ধোপররাধী } \\
\text { ফাঁসি }\end{array}$ & +0.520 & ভোদায় \\
\hline+1.389 & কোরআন & & $\begin{array}{l}\text { प्राग्य } \\
\text { ফাকिস্তান }\end{array}$ & $\begin{array}{l}+1.220 \\
+1219\end{array}$ & निজाমी & +0.530 & মৃত্যুদশ্ড \\
\hline+1.390 & খলিফা & & ইসরাইল & +1.215 & হেফাজতেইসনাম & $\begin{array}{l}+0.320 \\
+0.420\end{array}$ & $\begin{array}{l}\text { দালाা } \\
\text { জ|ফরবমাঁড }\end{array}$ \\
\hline+1.380 & জাহান্নাম & & काংলাদেশ & +1.115 & কাউয়াকদের & +0.214 & সুদখোর \\
\hline+1.260 & নরক & & পাকি & +1.112 & रनू & +0.150 & রাজাকার \\
\hline+1.242 & শিবির & & মোদী & +1.009 & ईলেকশন & +0.100 & চেতনাবাজ \\
\hline+1.213 & জামাত & & বিজেপি & +1.008 & निর্বাচন & +0.020 & গুম \\
\hline \multirow{2}{*}{\multicolumn{2}{|c|}{$\begin{array}{l}\ldots 1074 \text { more positive ... } \\
\ldots 15605 \text { more negative .. }\end{array}$}} & & ईज্ডিয়া & +1.007 & & +0.010 & জঙ্গি \\
\hline & & & ইরাক & \multirow{2}{*}{\multicolumn{2}{|c|}{$\begin{array}{l}\ldots 11007 \text { more positive } \ldots \\
\ldots 10772 \text { more negative } \ldots\end{array}$}} & \multirow{2}{*}{\multicolumn{2}{|c|}{$\begin{array}{l}11122 \text { more positive } \\
.22657 \text { more negative }\end{array}$}} \\
\hline-1.686 & বিবি & \multirow{2}{*}{\multicolumn{2}{|c|}{$\begin{array}{l}. .11710 \text { more positive } \ldots \\
.14069 \text { more negative } .\end{array}$}} & & & & \\
\hline-10.453 & সহবস্থান & & & -1.379 & মিছিল & \multirow{8}{*}{$\begin{array}{l}-0.852 \\
-0.894 \\
-1.181 \\
-1.243 \\
-1.242 \\
-1.241 \\
-1.230 \\
-1.221\end{array}$} & \\
\hline $\begin{array}{l}-10.455 \\
-10.550\end{array}$ & দৃগ্গাপুজা & -1.379 & ট্রানজিট & -1.200 & রাষ্ট্র & & ম|नু \\
\hline $\begin{array}{l}-10.550 \\
-10.600\end{array}$ & কালীপৃজা & -1.200 & & -1.191 & সরকার & & কলঙ \\
\hline-10770 & ঈদ & -1.191 & ফারাক্কা & -1.100 & পরিচালনা & & मारলा \\
\hline-10640 & তারাবাব & -1.100 & সামান্ত & -1.090 & नियन्तণ & & মেয়েরা \\
\hline-10.550 & রোজা & -1.090 & সুন্দরবন & -1.045 & |নিয়ত্র্রণ & & সत्रान \\
\hline-10340 & रस्दा & -1.090 & কাশ্মীর & -1.033 & উন্লয়न & & \\
\hline & নামাজ & -1.045 & বিএসএএফ & -0.976 & নিরাপত্তা & & ব্যাঙ্ক \\
\hline
\end{tabular}

Figure 8: Global feature importance, highlighting important terms per class

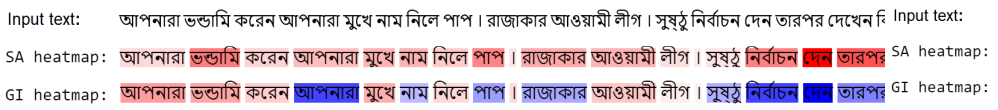

Input text: আপনারা ভন্ডামি করেন আপনারা মুখে নাম নিলে পাপ। রাজাকার আওয়ামী লীগ। স সুষৃঠু নির্বাচন দেন তারপর দেখেন f Input text:

SA heatmap : আপনারা ভন্ডামি করেন আপনারা মুখে নাম নিলে পাপ । রাজাকার আওয়ামী লীগ। সুষৃঠ্র নির্বাচন দেন তারপভ্ SA heatmap :

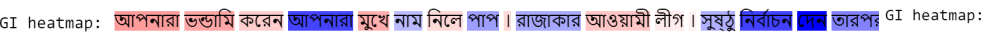

(a) Political hate

Input text: িক নাই দুধ দেখলে মনে হয় শুধু গুয়া মারছে না দুধে শুনা তরে এক হাত দন দিয়া গুয়া মারলে তখন

SA heatmap: টিক নাই দুধ দেখলে মনে হয় শুধু গুয়া মারছে না দুধে শুনা তরে এক হাত দন দিয়া গুয়া মারলে তখন GI heatmap: টিক নাই দুধ্ব দেখলে মনে হa্ শুধু গুয়া মারছে না দুধে শুনা তরে এক হাত দন দিয়া গুয়া মারলে তখন

(c) Personal hate
পুড়িয়ে হত্যা । মুসলিমদের ঘর বাড়ি আজও দখলে। মুসলিম জবাই হত্যা। বড় হারামজাদা জাতি হিন্দু মানাউনের বাচ্চারা। পুড়িয়ে হত্যা I মুসলিমদের ঘর বাড়ি আজও দখলে । মুসলিম জবাই হত্যা । বড় হারামজাদা জাতি হিন্দু মালাউনের বাচ্চারা ।

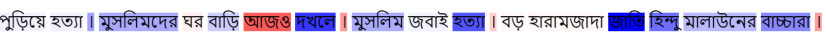

\section{(b) Religious hate}

Input text: ভারত আছে অুধু ওধু ফ্রি ট্রানজিট ফ্রি বন্দর সুবিধা ফ্রি ব্যান্ডউইথ নিতে। বিপদের বন্ধ চীন পাশে দাঁড়িয়েছে। ধন্যবাদ শি 3 শেখ হাসিনা।

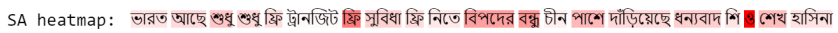

GI heatmap:

(d) Geopolitical hate

Figure 9: Example heat maps for for different types of hate, highlighting relevant terms

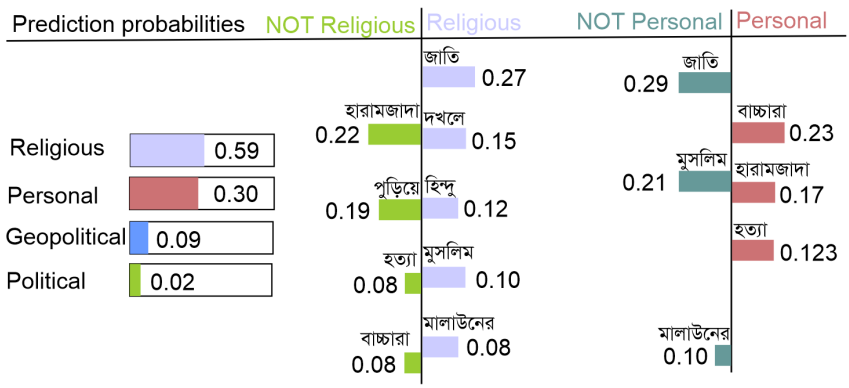

Figure 10: Word-level relevance test using leave-one-out

selection can have non-trivial impacts on learning capabilities of ML and DNN models, ii) even if a standalone ML and DNN baseline model does not perform well, the ensemble of several models may still outperform individual models.

Our approach has several potential limitations too. First, we had a limited amount of labelled data at hand during the training. Therefore, it would be unfair to claim that we could rule out the chance of overfitting. Secondly, we applied SA and LRP on a DNN baseline model (i.e., Conv-LSTM), albeit it would be more reasonable to do the same on the best performing standalone XLM-RoBERTa model. In future, we want to overcome these limitations by extending the datasets with a substantial amount of samples and applying SA and LRP on the XLM-RoBERTa model. Besides, we want to focus on other interesting areas such as named entity recognition, part-ofspeech tagging, sense disambiguation, and question answering for the Bengali language.

\section{REFERENCES}

[1] Mai Sherief, Vivek Kulkarni, and Elizabeth Belding. 2018. Hate lingo: a target-based linguistic analysis of hate speech in social media. In 12th AAAI Conference on Web and Social Media.

[2] Md Rezaul Karim, Bharathi Raja Chakravarthi, John P McCrae, and Michael Cochez. 2020. Classification benchmarks for under-resourced Bengali language based on multichannel convolutional-LSTM network. In 2020 IEEE 7th International Conference on Data Science and Advanced Analytics (DSAA). IEEE, 390-399. 
[3] Manoel Horta Ribeiro, Pedro H Calais, Virgílio AF Almeida, and Wagner Meira Jr. 2018. Characterizing and detecting hateful users on Twitter. In 12th AAAI conference on web and social media.

[4] A Guterres. 2019. United nations strategy and plan of action on hate speech. Taken from: https://www. un. org/en/genocidep 20Strategy.

[5] MS Islam. 2009. Research on Bangla language processing in Bangladesh: progress and challenges. In 8th International Language and Development Conference, 23-25.

[6] Ziqi Zhang, David Robinson, and Jonathan Tepper. 2018. Detecting hate speech on Twitter using a convolution-GRU based neural network. In ESWC. Springer, 745-760.

[7] R. Izs'ak. 2015. Hate speech and incitement to hatred against minorities in the media. UNHumans Rights Council, A/HRC/28/64

[8] Jacob Devlin, Ming-Wei Chang, Kenton Lee, and Kristina Toutanova. 2018. BERT: pre-training of deep bidirectional transformers for language understanding. arXiv:1810.04805.

[9] Yinhan Liu, Myle Ott, Naman Goyal, Mike Lewis, Luke Zettlemoyer, and Veselin Stoyanov. 2019. RoBERTa: a robustly optimized BERT pretraining approach. arXiv:1907.11692.

[10] Zhilin Yang, Zihang Dai, Yiming Yang, Jaime Carbonell, Russ R Salakhutdinov, and Quoc V Le. 2019. XLNET: generalized autoregressive pretraining for language understanding. In Advances in neural information processing systems, 57535763.

[11] Kevin Clark, Minh-Thang Luong, Quoc V Le, and Christopher D Manning. 2020. Electra: pre-training text encoders as discriminators rather than generators. arXiv:2003.10555.

[12] Sagor Sarker. 2020. Bangla-BERT: Bengali mask language model for Bengali language understading. (2020). https:// github.com/sagorbrur/bangla-bert.

[13] Joni Salminen, Hind Almerekhi, Milica Milenkovic, and Jung. 2018. Anatomy of online hate: developing a taxonomy and $\mathrm{ml}$ models for identifying and classifying hate in online news media. In ICWSM, 330-339.

[14] Nauros Romim, Mosahed Ahmed, Hriteshwar Talukder, and Md Saiful Islam. 2020. Hate speech detection in the bengali language: a dataset and its baseline evaluation. arXiv preprint arXiv:2012.09686.

[15] Alvi Md Ishmam and Sadia Sharmin. 2019. Hateful speech detection in public facebook pages for the Bengali language. In 2019 18th IEEE International Conference On Machine Learning And Applications (ICMLA). IEEE, 555-560.

[16] Brian Kenji Iwana, Ryohei Kuroki, and Seiichi Uchida. 2019. Explaining convolutional neural networks using softmax gradient layer-wise relevance propagation. arXiv:1908.04351.

[17] Andreas Holzinger, André Carrington, and Heimo Müller. 2020. Measuring the quality of explanations: the system causability scale (SCS). KI-Künstliche Intelligenz, 1-6.

[18] Binny Mathew, Punyajoy Saha, Seid Muhie Yimam, Chris Biemann, Pawan Goyal, and Animesh Mukherjee. 2020. Hatexplain: a benchmark dataset for explainable hate speech detection. arXiv preprint arXiv:2012.10289.

[19] Omar Zaidan, Jason Eisner, and Christine Piatko. 2007. Using annotator rationales to improve machine learning for text categorization. In Prof. of Human language technologies 2007:
The conference of the North American chapter of ACL, 260267.

[20] Andrea Saltelli. 2002. Sensitivity analysis for importance assessment. Risk analysis, 22, 3, 579-590.

[21] Bin Chen, Dennis Zaebst, and Lynn Seel. 2005. A macro ition/docutments/diate kappa statistics for categorizations by multiple raters. In Proceeding of the 30th Annual SAS Users Group International Conference. Citeseer, 155-30.

[22] Thierry Declerck and Piroska Lendvai. 2015. Processing and normalizing hashtags. In Proceedings of the International Conference Recent Advances in Natural Language Processing, 104109.

[23] Edouard Grave, Piotr Bojanowski, and Tomas Mikolov. 2018. Learning word vectors for 157 languages. In Proc. of the International Conference on Language Resources and Evaluation (LREC).

[24] Alexis Conneau, Kartikay Khandelwal, Naman Goyal, Vishrav Chaudhary, Guillaume Wenzek, Francisco Guzmán, Edouard Grave, Myle Ott, Luke Zettlemoyer, and Veselin Stoyanov. 2019. Unsupervised cross-lingual representation learning at scale. arXiv:1911.02116.

[25] Leila Arras, Grégoire Montavon, Klaus-Robert Muller, and Wojciech Samek. 2017. Explaining recurrent neural network predictions in sentiment analysis. arXiv:1706.07206.

[26] Jay DeYoung, Sarthak Jain, Nazneen Fatema Rajani, Eric Lehman, Caiming Xiong, Richard Socher, and Byron C Wallace. 2019. ERASER: a benchmark to evaluate rationalized nlp models. arXiv preprint arXiv:1911.03429.

[27] Elize Herrewijnen, Dong Nguyen, Jelte Mense, and Floris Bex. [n. d.] Machine-annotated rationales: faithfully explaining text classification.

[28] Charles H Martin and Michael W Mahoney. 2019. Traditional and heavy-tailed self regularization in neural network models. arXiv:1901.08276.

\section{APPENDIX}

Here, we provide more detail about baseline ML/DNN and transformer models. Further, to foster reproducibility, we make available the source codes, data, and interactive notebooks ${ }^{20}$. This repository will be updated with more reproducible resources, e.g., models, notebooks in the coming weeks.

\section{Training details for DNN baseline models}

The architectural parameters used to train a vanilla CNN, Bi-LSTM, and Conv-LSTM models are listed in Table 6, Table 7, and Table 8, respectively. The BiLSTM model is trained for 500 epochs. The idea is to observe how learning unfolds for each model and how the learning behaviour differs with bidirectional LSTM layers. The placement of bidirectional LSTM layers will create two copies of the hidden layer, one fit in the input sequences as is it is, while the second one on a reversed copy of the input sequence. This will make sure that the TimeDistributed layer ${ }^{21}$ receives 100 (or 300) timesteps of 32 outputs, instead of 10 timesteps of 64 (32 units +32 units) outputs. That is, the first hidden layer will have 100 memory

\footnotetext{
${ }^{20}$ https://github.com/rezacsedu/DeepHateExplainer

21 https://keras.io/api/layers/recurrent_layers/time_distributed/
} 
units, while the output layer will be a fully connected layer that outputs one value per timestep. The softmax activation function is used on the output to predict the types of hate. In other words, the output values from both BiLSTM layers will be concatenated, be fed into a fully connected softmax layer for the classification.

Table 6: Parameters for CNN model

\begin{tabular}{l|l}
\hline Parameter name & Parameter value \\
\hline Embedding dimension & $100,200,300$ \\
\hline Batch size & 32,64 \\
\hline CNN layer 1 & 64,128 \\
\hline CNN layer 2 & 32,64 \\
\hline Pooling size & 2,3 \\
\hline Dense layer 1 & 128,256 \\
\hline Dense layer 2 & 256,512 \\
\hline Dropout & $0.2,0.3$ \\
\hline Gaussian noise & $0.1,0.2,0.3,0.5$ \\
\hline Learning rate & $0.001,0.01,0.1$ \\
\hline
\end{tabular}

Table 7: Parameters for Bi-LSTM model

\begin{tabular}{l|l}
\hline Parameter name & Parameter value \\
\hline Embedding dimension & $100,200,300$ \\
\hline Batch size & 32,64 \\
\hline Bidirectional LSTM layer 1 & 32,64 \\
\hline Bidirectional LSTM layer 2 & 32,64 \\
\hline Dense layer 1 & 128,256 \\
\hline Dense layer 2 & 256,512 \\
\hline Dropout & $0.2,0.3$ \\
\hline Gaussian noise & $0.1,0.2,0.3,0.5$ \\
\hline Learning rate & $0.001,0.01,0.1$ \\
\hline
\end{tabular}

Table 8: Parameters for Conv-LSTM model

\begin{tabular}{l|l}
\hline Parameter name & Parameter value \\
\hline Embedding dimension & $100,200,300$ \\
\hline Batch size & 32,64 \\
\hline CNN layer 1 & 64,128 \\
\hline CNN layer 2 & 32,64 \\
\hline Pooling size & 2,3 \\
\hline LSTM layer 1 & 32,64 \\
\hline LSTM layer 2 & 32,64 \\
\hline Dense layer 1 & 128,256 \\
\hline Dense layer 2 & 256,512 \\
\hline Dropout & $0.2,0.3$ \\
\hline Gaussian noise & $0.1,0.2,0.3,0.5$ \\
\hline Learning rate & $0.001,0.01,0.1$ \\
\hline
\end{tabular}

During the training of Conv-LSTM, an LSTM layer treats an input feature space of $100 \times 300$ and its embedded feature vector dimension as timesteps, which generates 100 hidden units per timestep. Once the embedding layer passes an input feature space $100 \times 300$ into a convolutional layer, the input is padded such that the output has the same length as the original input. Then the output of each convolutional layer is passed to the dropout (or Gaussian noise) layer to regularize learning to avoid overfitting. This involves the input feature space into a $100 \times 100$ representation, which is then further down-sampled by three different 1D max-pooling layers, each having a pool size of 4 along with the word dimension, each producing an output of shape $25 \times 100$, where each of 25 dimensions can be considered as extracted features. Each max-pooling layer follows to flatten the output space by taking the highest value in each timestep dimension, which produces a $1 \times 100$ vector that forces words that are highly indicative of interest. These vectors are then fed into a fully connected softmax layer to predict the probability distribution over the hate classes.

\section{Training details for ML baseline models}

We train LR, SVM, KNN, NB, RF, and GBT ML baselines models using the scikit-learn library. We apply both character n-grams and word uni-grams with TF-IDF weighting. The best hyperparameters are produced through random and with 5 -fold cross-validation tests. More specifically, Fig. 11 listed the hyperparameters considered in a random search setting.

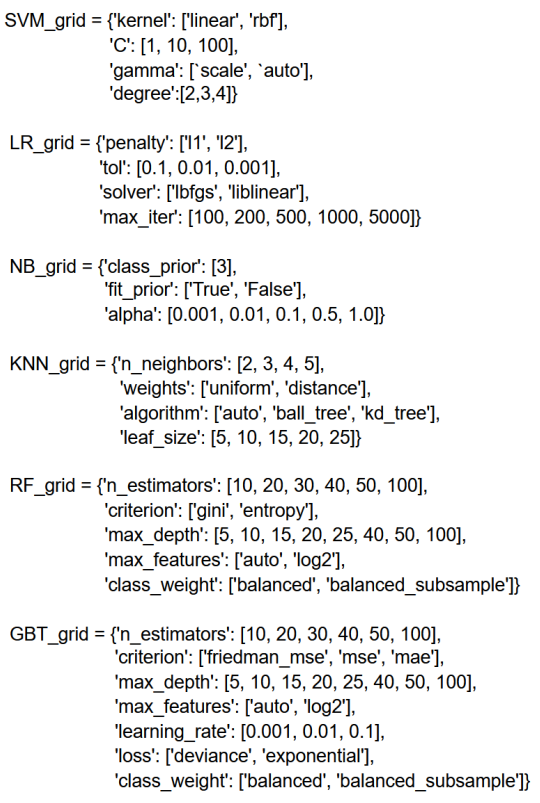

Figure 11: Param grids for ML base line models

\section{Classification results}

We enlist evaluation results of each BERT variant and ensemble of top models on the held-out test set, where the following class encoding to interpret the class-specific classification: i) personal hate: class 0 , ii) political hate: class 1 , iii) religious hate: class 2 , and iv) geopolitical hate: class 3 . We provide class-wise classification result for each BERT variant, while the same based on the ensemble prediction is shown in Table 4, covering each hate category.

\section{Explanations}

We provide two examples that highlight important terms a DNN model puts more attention to. The example ${ }^{22}$ in fig. 16 , shows positive feature importances represent the extent that the word was important towards the classification of the selected label, while negative feature importances represents words that encouraged the model away from the selected label. Either positive, negative or both positive and negative features can be selected, outlining their relative importance, while ?? shows an example ${ }^{23} \mathrm{de}-$ tection of personal hate based on using SA, LRP, and integrated gradients (GI). LRP accurately highlights (deep blue) most relevant

\footnotetext{
22 https://github.com/rezacsedu/DeepHateExplainer/blob/main/notebooks/Example_interpret_text.ipynb 23 https://github.com/rezacsedu/DeepHateExplainer/blob/main/notebooks/LRP_BiLSTM_FastText_Embb
} 


\begin{tabular}{|c|c|c|c|c|}
\hline \multicolumn{5}{|c|}{ Accuracy: 0.8675} \\
\hline \multicolumn{5}{|c|}{ Precision: 0.8696318194488171} \\
\hline \multicolumn{5}{|c|}{ Recall: 0.8675} \\
\hline \multicolumn{5}{|c|}{ F1-score: 0.867557867752591} \\
\hline \multicolumn{5}{|c|}{ MCC: 0.8080296942576948} \\
\hline \multicolumn{5}{|c|}{ Class-wise classification report: } \\
\hline & precision & recall & f1-score & support \\
\hline 0 & 0.93 & 0.90 & 0.91 & 524 \\
\hline 1 & 0.79 & 0.72 & 0.75 & 157 \\
\hline 2 & 0.77 & 0.90 & 0.83 & 159 \\
\hline 3 & 0.87 & 0.88 & 0.87 & 360 \\
\hline accuracy & & & 0.87 & 1200 \\
\hline macro avg & 0.84 & 0.85 & 0.84 & 1200 \\
\hline weighted avg & 0.87 & 0.87 & 0.87 & 1200 \\
\hline
\end{tabular}

Figure 15: Class-wise classification results based on XLM-RoBERTa model

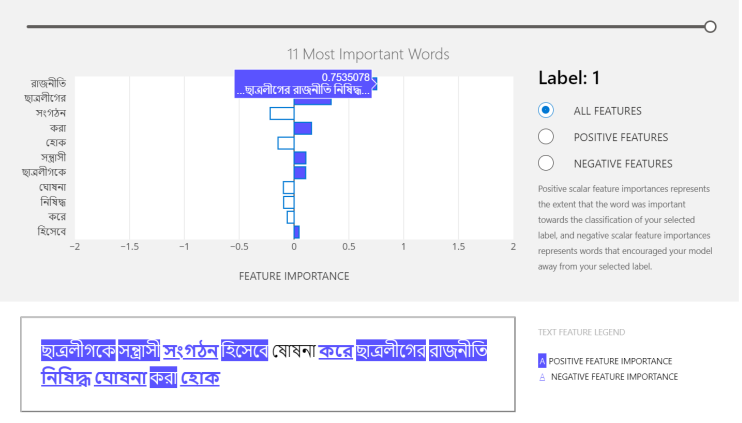

Figure 16: Example-1: identification of political hate, showing most relevant terms

Predicted lab
LRP heatmap:

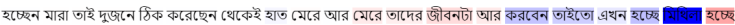

A heatmap:

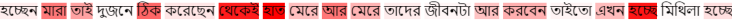
GI heatmap:

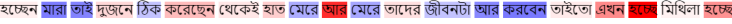

Figure 17: Example-2: identification of personal hate, showing most relevant terms

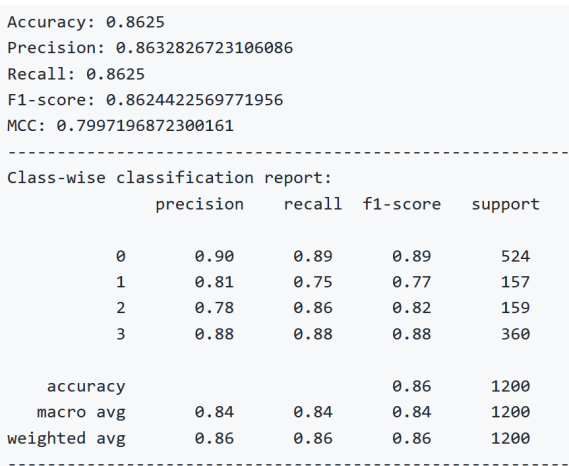

Figure 12: Class-wise classification results based on Bangla-BERT model

\begin{tabular}{|c|c|c|c|c|}
\hline \multirow{2}{*}{\multicolumn{5}{|c|}{ Accuracy: 0.8466666666666667}} \\
\hline & & & & \\
\hline \multicolumn{5}{|c|}{ Precision: 0.8453902573996248} \\
\hline \multicolumn{5}{|c|}{ Recall: 0.8466666666666667} \\
\hline \multicolumn{5}{|c|}{ F1-score: 0.8453045673170627} \\
\hline \multicolumn{5}{|c|}{ MCC: $\theta .7747627586951363$} \\
\hline \multicolumn{5}{|c|}{ Class-wise classification report: } \\
\hline & precision & recall & f1-score & suppor \\
\hline 0 & 0.87 & 0.91 & 0.89 & 524 \\
\hline 1 & 0.79 & 0.69 & 0.73 & 157 \\
\hline 2 & 0.82 & 0.84 & 0.83 & 159 \\
\hline 3 & 0.85 & 0.83 & 0.84 & 360 \\
\hline accuracy & & & 0.85 & 1200 \\
\hline macro avg & 0.83 & 0.82 & 0.82 & 1200 \\
\hline weighted avg & 0.85 & 0.85 & 0.85 & 1200 \\
\hline
\end{tabular}

Figure 13: Class-wise classification results based on BERT-base-multilingual-cased model

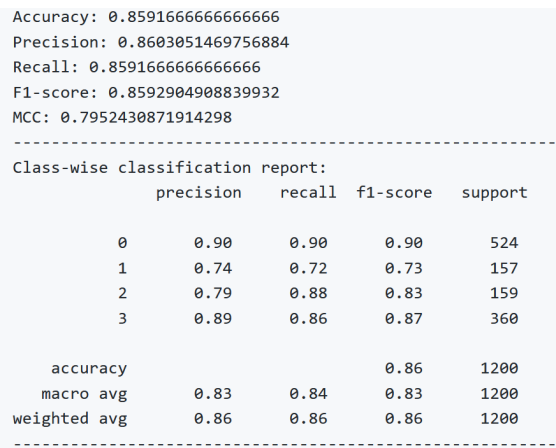

Figure 14: Class-wise classification results based on BERT-base-multilingual-uncased model

words মिथिলा (Bangladeshi actress Mithila), which signify a personal hate. 